\title{
Outcomes of Extended Endovascular Aortic Repair for Aorto-Iliac Aneurysm with Internal Iliac Artery Occlusion
}

\author{
Shunichiro Fujioka, MD, ${ }^{1}$ Shigeru Hosaka, MD, PhD, ${ }^{1}$ Hayato Morimura, MD, ${ }^{1}$ \\ Ken Chen, MD, ${ }^{1}$ Zhi Chao Wang, MD, ${ }^{1}$ Koji Toguchi, MD, ${ }^{1}$ Shoji Fukuda, MD, PhD, ${ }^{1}$ \\ Koki Takizawa, MD, ${ }^{2}$ and Hiroshi Osawa, MD, PhD²
}

\begin{abstract}
Objective: Patients of aorto-iliac aneurysms who undergo endovascular aortic repair (EVAR) require internal iliac artery (IIA) occlusion with coil embolization and its coverage with the stent graft to prevent type II endoleak after extending the endograft into the external iliac artery. However, it has become well recognized that IIA occlusion cause buttock claudication and other various sequelae due to pelvic ischemia. We retrospectively analyzed IIA occlusion outcomes.

Methods: From October 2008 to February 2015, 71 patients with aorto-iliac aneurysms underwent IIA occlusion prior to EVAR. The relationship between pelvic circulation and symptom of pelvic ischemia was studied.
\end{abstract}

Results: Buttock claudication occurred in 17 patients (22.9\%) of all. Eight patients (14.8\%) in unilateral IIA occlusion group (54 patients) and nine patients (52.9\%) in bilateral IIA group (17 patients) had sequelae of claudication. The sacrifice of the communication of superior gluteal artery (SGA) and inferior gluteal artery (IGA) led to buttock claudication in $18(64.3 \%)$ of 28 limbs. Instead, only 4 of 60 limbs had buttock claudication, when we preserved the communication between SGA and IGA. In all patients, staged treatment of aorto-iliac aneurysms with IIA occlusion and EVAR were done successfully without pelvic ischemic complications except for buttock claudication, and postoperative CT scanning showed no endoleakage.

Conclusion: IIA occlusion prior to EVAR is recognized as a safe and reasonable strategy. It is emphasized that preservation of the communication of SGA and IGA is important to prevent buttock claudication. (This is a translation of Jpn J Vasc Surg 2016; 25: 240-245.)

\footnotetext{
${ }^{1}$ Department of Cardiovascular Surgery, National Center for Global Health and Medicine, Tokyo, Japan

${ }^{2}$ Department of Cardiovascular Surgery, Shimada General Hospital, Choshi, Chiba, Japan

Received: August 18, 2017; Accepted: August 18, 2017 Corresponding author: Shunichiro Fujioka, MD. Department of Cardiovascular Surgery, National Center for Global Health and Medicine, 1-21-1 Toyama, Shinjuku, Tokyo 162-8655, Japan Tel: +81-3-3202-7181, Fax: +81-3-3202-7364

E-mail: winter_solstice_s@yahoo.co.jp

This is a translation of Jpn J Vasc Surg 2016; 25: 240-245.
}

Keywords: endovascular aortic repair, iliac artery coil embolization, buttock claudication

\section{Introduction}

Recently, endovascular aortic repair (EVAR) has been established as a minimally invasive surgical procedure for abdominal aortic aneurysm (AAA) repair. Favorable results have been reported for EVAR performed in accordance with the Instructions For Use (IFU) described in the package insert of the stent graft. The IFU states that the procedure is not indicated for lesions in the bilateral external iliac arteries.

On the other hand, $40 \%$ of AAA cases were reported to be complicated by unilateral or bilateral iliac artery aneurysms. ${ }^{1)}$ The scope of EVAR indications will be expanded by adding treatment of the internal iliac artery, particularly coil embolization, to prevent type II endoleak in such complicated cases.

However, buttock claudication after coil embolization of the internal iliac artery is a complication in some cases. ${ }^{2)}$ We considered that collateral blood circulation from the deep femoral artery via the inferior gluteal artery is deeply involved in the occurrence of such cases of buttock claudication. We attempted to prevent buttock claudication by preserving the communication between the superior and inferior gluteal arteries (superior-inferior gluteal communication) as much as possible during coil embolization of the internal iliac artery and by performing EVAR within a certain time interval after coil embolization to facilitate the development of collateral blood circulation.

In this study, we evaluated the safety of EVAR combined with coil embolization of the internal iliac artery, including coil embolization of the bilateral internal iliac arteries, particularly in terms of the incidence of buttock claudication. 


\section{Subjects}

From October 2008 to February 2015, EVAR was performed in 165 patients with AAA who were determined to be ineligible for open aortic graft replacement because of advanced age, history of open surgery, or other reasons. Of these patients, 71 were deemed to require landing in the external iliac artery based on three-dimensional computed tomography (CT) measurements. They underwent internal iliac artery occlusion by coil embolization or some other methods to prevent type II endoleak and received a stent graft reaching the external iliac artery. These 71 patients were included in this study.

\section{Methods}

For patients undergoing scheduled surgery, internal iliac artery occlusion was performed 2 weeks before EVAR via coil embolization in principle using the contralateral femoral artery approach with the patient under local anesthesia. For patients who underwent emergency surgery, internal iliac artery occlusion was performed with singlestage EVAR. EVAR combined with bilateral internal iliac artery occlusion was performed in three stages with a 2-week interval between each component.

An auxiliary device capable of occluding the internal iliac artery bifurcation was selected, and the internal iliac artery was occluded with the main unit of the device when coil embolization was unsuccessful due to anatomical or other factors or when the peripheral side of the common iliac artery in the vicinity of the internal iliac artery bifurcation was equivalent in diameter to that of the external iliac artery and direct occlusion of the internal iliac artery bifurcation with a stent graft appeared possible. ${ }^{3,4)}$

During coil embolization, we placed a coil on the proximal side of the internal iliac artery as much as possible in patients with a concomitant common iliac artery aneurysm and tried not to affect the bifurcation of the superior and inferior gluteal arteries to preserve the superior-inferior gluteal communication. For patients with a concomitant internal iliac artery aneurysm, embolization was achieved by placing a coil on the distal side of the aneurysm, or when the internal iliac artery aneurysm had advanced to reach the bifurcation of the superior and inferior gluteal arteries, by placing a coil in the aneurysm to embolize the branches from the aneurysm.

In all patients, a commercially available stent graft was used for EVAR, which was inserted via the bilateral common femoral arteries with the patient under general anesthesia.

Contrast CT was performed 1 and 6 months and 1 year after surgery and every year thereafter to assess changes in aneurysm diameter and for the presence of endoleak.
Patients were also asked if they had buttock claudication at the same time points.

Statistics are expressed as mean \pm standard deviation. For statistical analysis, the $\chi^{2}$ and Mann-Whitney U tests were used, and a difference was considered significant when $\mathrm{P}<0.05$.

\section{Results}

The 71 patients (mean age, 75 years; range, $60-91$ years) studied included 56 men $(79 \%)$ and 15 women $(21 \%)$. Iliac artery occlusion was performed on 88 limbs, unilaterally in 54 (unilateral occlusion group) and bilaterally in 17 (bilateral occlusion group). Methods for internal iliac artery occlusion were coil embolization in 65 patients and direct occlusion with a stent graft in 6. Devices used were the Excluder (W. L. Gore \& Associates, USA) in 33 patients (46\%), Endurant (Medtronic, USA) in $17(24 \%)$, and Powerlink (Endologix, USA) in $21(30 \%)$.

Buttock claudication associated with internal iliac artery occlusion occurred in 17 patients (22 limbs), including $8 / 54$ (14.8\%; 8 limbs) in the unilateral and 9/17 $(52.9 \% ; 14$ limbs) in the bilateral occlusion groups, which were significantly different $(\mathrm{P}=0.0034)$.

Age, body weight (BW), and body mass index (BMI) of patients with and without postoperative buttock claudication were $72.3 \pm 9.5$ versus $76.0 \pm 7.7$ years, $64.7 \pm 11.6$ versus $58.1 \pm 12.2 \mathrm{~kg}$, and $24.2 \pm 3.2$ versus $22.1 \pm 4.0$, respectively, indicating that the incidence of complication with buttock claudication tended to be higher in younger patients with greater BW and that the BMI was significantly higher in those with buttock claudication (Table 1).

However, the superior-inferior gluteal communication could not be preserved due to an internal iliac aneurysm or procedural complications in $15 / 54(27.8 \%)$ and $8 / 17$ $(57.8 \%)$ patients (13/34 limbs, $38.2 \%)$, respectively.

In the unilateral occlusion group, buttock claudication occurred in only $2 / 39$ patients $(5 \%)$ with successful preservation of the superior-inferior gluteal communication compared with $6 / 15$ patients $(40 \%)$ with unsuccessful preservation. The incidence was significantly higher in the latter subgroup (Fig. 1). In the bilateral occlusion group,

Table 1 Patients' characteristics

\begin{tabular}{lccc}
\hline & $\begin{array}{c}\text { Buttock } \\
\text { claudication } \\
(+)\end{array}$ & $\begin{array}{c}\text { Buttock } \\
\text { claudication } \\
(-)\end{array}$ & P value \\
\hline Patients number & 17 & 54 & \\
Age (year) & $72.3 \pm 9.5$ & $76.0 \pm 7.7$ & 0.055 \\
BW (kg) & $64.7 \pm 11.6$ & $58.1 \pm 12.2$ & 0.056 \\
BMl & $24.2 \pm 3.2$ & $22.1 \pm 4.0$ & 0.046 \\
\hline
\end{tabular}

BW: body weight; BMI: body mass index 


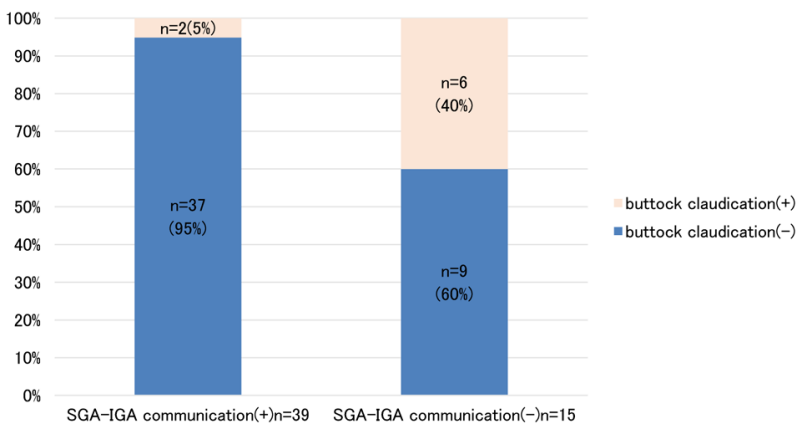

Fig. 1 Relationship between the complication of buttock claudication and SGA-IGA communication of unilateral occlusion group. SGA: superior gluteal artery; IGA: inferior gluteal artery

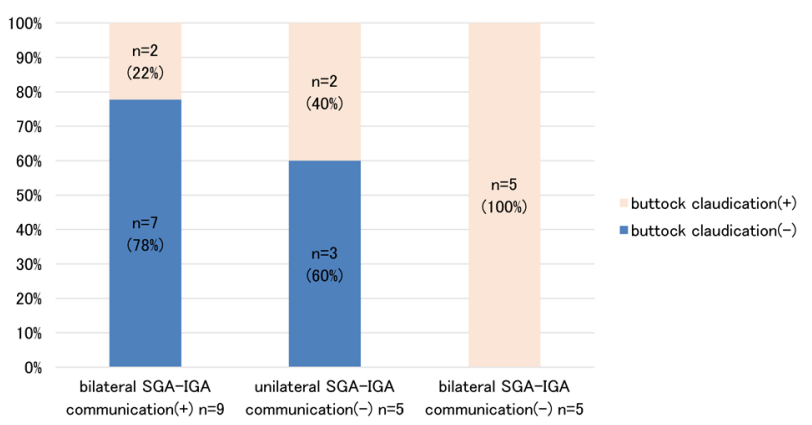

Fig. 2 Relationship between the complication of hip claudication and SGA-IGA communication of bilateral occlusion group. SGA: superior gluteal artery; IGA: inferior gluteal artery

buttock claudication occurred in 2/9 (22\%), 2/5 (40\%), and $5 / 5(100 \%)$ patients with successful preservation of the communication on both sides, only one side, and neither side, respectively. The incidence of buttock claudication tended to increase in the latter two subgroups (Fig. 2).

When the association between the preservation status of the superior-inferior gluteal communication and incidence of buttock claudication was assessed in all 88 limbs treated with internal iliac artery occlusion, buttock claudication was noted in $4 / 60$ limbs $(7 \%)$ with successful preservation of the communication, whereas the incidence increased to $18 / 28 \mathrm{limbs}(64 \%)$ with successful preservation of the communication.

When these 88 limbs were analyzed with unilateral versus bilateral occlusion as an additional stratification factor, buttock claudication was noted in $2 / 39(5 \%), 6 / 15$ (40\%), 2/21 (10\%), 12/13 (92\%), and 12/15 (80\%) limbs with unilateral occlusion and successful preservation, unilateral occlusion and unsuccessful preservation, bilateral occlusion and successful preservation, bilateral occlusion and unilateral unsuccessful preservation, and unsuccessful preservation on both sides, respectively (Fig. 3).

Buttock claudication occurred after internal iliac artery occlusion, such as coil embolization, but disappeared in

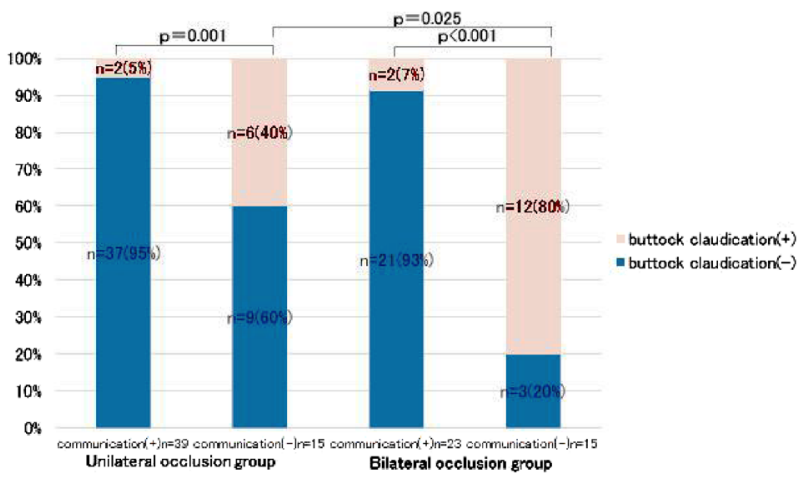

Fig. 3 Relationship of the complication of hip claudication with SGA-IGA communication and contralateral SGA-IGA communication in all cases. SGA: superior gluteal artery; IGA: inferior gluteal artery

Table 2 Duration of hip claudication

\begin{tabular}{llc}
\hline & & $\begin{array}{c}\text { Days } \\
\text { (mean) }\end{array}$ \\
\hline Unilateral occlusion group & communication (+) & 105 \\
& communication (-) & 209 \\
Bilateral occlusion group & Bilateral communication (+) & 22 \\
& Unilateral communication (+) & 37 \\
& communication (-) & 275 \\
\hline
\end{tabular}

all cases within 1 year of EVAR. The mean duration was $105,209,22,37$, and 275 days in cases with unilateral occlusion and successful preservation of the communication, unilateral occlusion and unsuccessful preservation, bilateral occlusion and successful preservation on both sides, bilateral occlusion and successful preservation only on one side, and bilateral occlusion and unsuccessful preservation on any side, respectively (Table 2 ).

In patients with buttock claudication due to coil embolization performed before EVAR as planned, no further symptom exacerbation was observed after EVAR.

Patients were evaluated with contrast CT at 1 and 6 months and 1 year postoperatively. Type II endoleak from the internal iliac artery was not observed in the arterial or venous phase in any patient. Perioperative deaths or symptoms suggestive of intestinal ischemia were also not observed, and there were no complaints of new sexual dysfunction or pelvic viscera dysfunction, such as urinary retention.

\section{Discussion}

An extended AAA often advances to the common iliac or even the internal iliac artery to form a series of aneurysm or noncontinuous, isolated aneurysms of the common iliac or internal iliac artery. Such unilateral or bilateral iliac artery aneurysms have been reported in $40 \%$ of pa- 
tients with AAAs. ${ }^{1)}$ In addition to conventional EVAR, endovascular aortic repair in such cases is required for the treatment of the internal iliac artery to prevent type II endoleak.

However, it has been noted that pelvic viscera ischemia or buttock claudication can occur even with unilateral internal iliac artery coil embolization..$^{5)}$ To prevent this, coil embolization at the proximal internal iliac artery has been reported to preserve the superior-inferior gluteal communication and reduce the incidence of buttock claudication. ${ }^{6,7)}$ In our cases too, the incidence of buttock claudication in patients with unsuccessful superior-inferior gluteal communication preservation was $64 \%$, which is higher than the $7 \%$ observed in patients with successful preservation.

When the unilateral versus bilateral occlusion groups were compared, the incidence of buttock claudication was higher in the latter group ( $14.8 \%$ versus $52.9 \%)$. This difference was understandable as pelvic viscera perfusion routes available after coil embolization of the bilateral internal iliac arteries are mainly limited to collateral blood circulation from the ipsilateral inferior mesenteric, lumbar, superficial iliac circumflex (a branch of the external iliac artery), and deep femoral arteries, whereas collateral blood circulation from the contralateral internal iliac artery is preserved in the unilateral embolization group.

An even greater difference in the incidence of buttock claudication was observed between cases with successful versus unsuccessful preservation of the superior-inferior gluteal communication ( $7 \%$ versus $64 \%$ ). As shown in Fig. 3, the comparison among the 88 limbs stratified according to unilateral/bilateral occlusion and the preservation status of the superior-inferior gluteal communication indicated that preserved communication is key to prevent buttock claudication and that collateral blood circulation from the ipsilateral deep femoral artery via the inferior gluteal artery is critical for the pelvic viscera, particularly for the superior gluteal artery region served by gluteal muscle blood flow.

This result suggested that deep femoral arterioplasty performed during EVAR can be an effective means to reverse buttock claudication occurring after internal iliac artery occlusion in patients who preoperatively presented with an advanced stenotic lesion at the origin of the deep femoral artery.

It also was found that the incidence of buttock claudication tended to be higher in young patients with high $\mathrm{BW}$ and BMI. This was considered to be because young patients have high physical activity and patients with high BMI, who have a strong tendency to be obese, are subjected to BW burden so that they are prone to suffer buttock claudication. Therefore, we believe that more careful efforts to preserve the superior-inferior gluteal communication are necessary for these patient subgroups.

Coil embolization of the internal iliac artery was not selected and the bifurcation was directly embolized using a stent graft in patients in whom coil embolization was deemed difficult due to anatomical factors, such as an extremely thin internal iliac artery bifurcation and the presence of irregular atheroma around the origin, or there was virtually no difference in diameter between the proximal external iliac artery and bifurcation of the internal iliac artery. This procedure was performed in six patients, and satisfactory closure of the internal iliac artery was achieved. This appears to be a useful method that can simplify the surgical procedure and ensure preservation of the superior-inferior gluteal communication. We also believe that this procedure is useful to reduce radiation exposure to patients and surgeons in cases where coil embolization is predicted to be extremely difficult.

In addition, the number of coils used for embolization sometimes becomes an issue due to the current high medical cost. At our hospital, the mean number of coils used per side was 7 (4-11). Direct closure of the bifurcation of the internal iliac artery using a stent graft, as described above, does not require any coil for embolization, and we believe that it also is useful for cost reduction. Regarding the cost-effectiveness of EVAR and open surgery, EVAR has previously been reported to be more expensive than open surgery, ${ }^{81}$ but a recent study has shown that EVAR is cost-effectiveness. 9) Our strategy of EVAR for aortoiliac aneurysm, EVAR requires a total of 12-15 days of hospitalization ( 3 days for coil embolization and 9 days for EVAR), which is comparable to the mean length of hospital stay in patients who underwent open aortic graft replacement at our hospital (average 14 days), even when bilateral coil embolization was performed.

In any case, we believe that safe and complication-free treatment of patients in whom open surgery is difficult is important from the viewpoint of cost-effectiveness.

We usually schedule coil embolization and EVAR to be performed with a 2-week or longer interval, but we cannot recommend how long this interval should be based on our study. In the EVAR strategy that closes the internal iliac artery, collateral blood circulation from the inferior mesenteric and lumbar arteries is eventually blocked, and thus, the degree of ischemia in the internal iliac artery perfusion area is expected to be appreciably greater when EVAR is completed than when coil embolization is completed. However, we have never encountered a case wherein buttock claudication was exacerbated after EVAR. This may suggest that a 2 -week interval is reasonable as a period to promote collateral blood circulation. Furthermore, a single-stage operation including coil embolization and EVAR requires longer duration of anesthesia and greater amount of contrast agent during surgery and is not pref- 
erable for patients with reduced renal function. Thus, we believe that the significance of separately performing these procedures is high in terms of invasiveness.

In addition, intestinal ischemia, the most serious complication associated with internal iliac artery occlusion, was not observed in our 54 patients (88 limbs) who underwent EVAR combined with internal iliac artery occlusion. This suggested that the glutei are most susceptible to ischemia due to internal iliac artery occlusion and that they have a greater oxygen demand and are more readily affected by reduced perfusion pressure and amount than the pelvic viscera, such as the rectum and sigmoid colon. Thus, the incidence of buttock claudication may serve as an excellent clinical indicator that reflects pelvic viscera ischemia as the only disadvantage of this surgical procedure.

\section{Conclusion}

Internal iliac artery occlusion can be safely performed with coil embolization during EVAR. However, preserving the superior-inferior gluteal communication is critically important to prevent buttock claudication by maintaining collateral blood circulation from the deep femoral artery.

\section{Disclosure Statement}

All authors and co-authors have no conflict of interest.

\section{References}

1) Hobo R, Sybrandy JEM, Harris P, et al.; EUROSTAR Collaborators. Endovascular repair of abdominal aortic aneurysms with concomitant common iliac artery aneurysm. Outcome analysis of the EUROSTAR experience. J Endovasc Ther 2008; 15: 12-22.

2) Razavi MK, DeGroot M, Olcott C 3rd, et al. Internal iliac artery embolization in the stent-graft treatment of aortoiliac aneurysms: analysis of outcomes and complications. J Vasc Interv Radiol 2000; 11: 561-6.

3) Papazoglou KO, Sfyroeras GS, Zambas N, et al. Outcomes of endovascular aneurysm repair with selective internal iliac artery coverage without coil embolization. J Vasc Surg 2012; 56: 298-303.

4) Bharwani N, Raja J, Choke E, et al. Is internal iliac artery embolization essential prior to endovascular repair of aortoiliac aneurysms? Cardiovasc Intervent Radiol 2008; 31 : 504-8.

5) Ghosh J, Murray D, Paravastu S, et al. Contemporary management of aorto-iliac aneurysms in the endovascular era. Eur J Vasc Endovasc Surg 2009; 37: 182-8.

6) Kritpracha B, Pigott JP, Price CI, et al. Distal internal iliac artery embolization: a procedure to avoid. J Vasc Surg 2003; 37: 943-8.

7) Bratby MJ, Munneke GM, Belli AM, et al. How safe is bilateral internal iliac artery embolization prior to EVAR? Cardiovasc Intervent Radiol 2008; 31: 246-53.

8) The United Kingdom EVAR Trial Investigator. Endovascular versus open repair of abdominal aortic aneurysm. N Engl J Med 2010; 362: 1863-71.

9) Burgers LT, Vahl AC, Severens JL, et al. Cost-effectiveness of elective endovascular aneurysm repair versus open surgical repair of abdominal aortic aneurysms. Eur J Vasc Endovasc Surg 2016; 52: 29-40. 